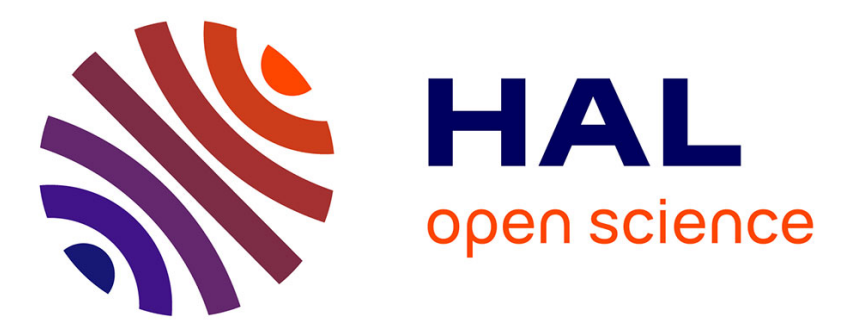

\title{
Semiautomatic quantification of abdominal wall muscles deformations based on dynamic MRI image registration
}

Arthur Jourdan, Arnaud Le Troter, Pierre Daude, Stanislas Rapacchi, Catherine Masson, Thierry Bège, David Bendahan

\section{To cite this version:}

Arthur Jourdan, Arnaud Le Troter, Pierre Daude, Stanislas Rapacchi, Catherine Masson, et al.. Semiautomatic quantification of abdominal wall muscles deformations based on dynamic MRI image registration. NMR in Biomedicine, In press, 10.1002/nbm.4470 . hal-03145836

\author{
HAL Id: hal-03145836 \\ https://hal.science/hal-03145836
}

Submitted on 18 Feb 2021

HAL is a multi-disciplinary open access archive for the deposit and dissemination of scientific research documents, whether they are published or not. The documents may come from teaching and research institutions in France or abroad, or from public or private research centers.
L'archive ouverte pluridisciplinaire HAL, est destinée au dépôt et à la diffusion de documents scientifiques de niveau recherche, publiés ou non, émanant des établissements d'enseignement et de recherche français ou étrangers, des laboratoires publics ou privés. 


\title{
SEMI-AUTOMATIC QUANTIFICATION OF ABDOMINAL WALL MUSCLES DEFORMATIONS BASED ON DYNAMIC MRI IMAGE REGISTRATION
}

\author{
Arthur Jourdan $_{a}$, Arnaud Le Troter $_{b}$, Pierre Daude ${ }_{b}$, Stanislas Rapacchi ${ }_{b}$, Catherine \\ Masson $_{a}$, Thierry Bège $_{a, c}$, David Bendahan $b$ \\ a. Aix-Marseille Univ, Univ Gustave Eiffel, IFSTTAR, LBA, F-13016 Marseille, France \\ b. Aix Marseille Univ, CNRS, CRMBM, Marseille, France \\ c. Department of General Surgery, Aix Marseille Univ, North Hospital, APHM, Marseille, France
}

\section{Corresponding author}

Arthur Jourdan : arthur.jourdan@univ-eiffel.fr

Université Gustave Eiffel, Faculté de Médecine-Secteur Nord, Boulevard P. Dramard, 13916, Marseille Cedex 20, France.

\section{E-mail adresses}

Arnaud le Troter : arnaud.le-troter@univ-amu.fr

Pierre Daude : pierre.daude@univ-amu.fr

Stanislas Rapacchi : stanislas.rapacchi@univ-amu.fr

Catherine Masson : catherine.masson@univ-eiffel.fr

Thierry Bège : thierry.bege@ap-hm.fr

David Bendahan : david.bendahan@univ-amu.fr

\section{Keywords}

Abdominal wall, Dynamic magnetic resonance imaging, Segmentation, Image registration, Deformations, Parcellation

\begin{abstract}
Abbreviations
WC : waist circumference; RRA : right rectus abdominis; LRA : left rectus abdominis; RLM : right lateral muscles; LLM : left lateral muscles; PM : posterior abdominal muscles; AO : aorta; VI : Viscera; SF : subcutaneous; HD : Hausdorff distance

\section{Data availability statement}

The data that support the findings of the present study can be made available to experts upon request and their use will be conditioned by the signature of a material transfer agreement.
\end{abstract}




\section{Abstract summary (273 words)}

Quantitative analysis of abdominal organs motion and deformation is crucial to better understand biomechanical alterations undermining respiratory, digestive or perineal pathophysiology. In particular, biomechanical characterization of the antero-lateral abdominal wall is central in the diagnostic of abdominal muscle deficiency. We present here a dedicated semi-automatic dynamic MRI post-processing method enabling the quantification of spatial and temporal deformations of the antero-lateral abdominal wall muscles.

10 healthy participants were imaged during a controlled breathing session at the L3-L4 disc level using real-time dynamic MRI at 3T. A coarse feature tracking step allowed the selection of the inhalation cycle of maximum abdominal excursion. Over this image series, the described method combines i) a supervised $2 \mathrm{D}+\mathrm{t}$ segmentation procedure of the abdominal wall muscles, ii) the quantification of muscles deformations based on masks registration and iii) the mapping of deformations within muscle sub-zones leveraging a dedicated automatic parcellation.

The supervised $2 \mathrm{D}+\mathrm{t}$ segmentation (i) provided an accurate segmentation of the abdominal wall muscles throughout maximum inhalation with a $0.95 \pm 0.03$ DSC value and a $2.3 \pm 0.7 \mathrm{~mm}$ Hausdorff distance value while requiring only manual segmentation of $20 \%$ of the data. The robustness of the deformation quantification (ii) was indicated by high indices of correspondence between the registered source mask and the target mask $(0.98 \pm 0.01$ DSC value and $2.1 \pm 1.5 \mathrm{~mm}$ Hausdorff distance value). Parcellation (iii) enabled the distinction of muscle substructures which are anatomically relevant but could not be distinguished based on images contrast.

The present genuine post-processing method provides a quantitative analytical frame that could be used in further studies for a better understanding of abdominal wall deformations in physiological and pathological situations. 


\section{Introduction}

Real-time dynamic MRI is a promising modality in the field of medical imaging given its capacity to capture organs motion and deformation. It is currently used for the diagnosis of cardiac dysfunction and perfusion ${ }^{1,2}$ and pelvis organ prolapse ${ }^{3}$. This diagnostic approach is still often limited to a qualitative analysis of images by experts whereas quantitative information would enable to refine it. In particular, quantitative biomechanical characterization from realtime MRI would need to be provided to clinicians in synthetic formats so that their medical decision can be supported by robust and self-explained quantitative methods ${ }^{4,5}$.

Although of interest, kinetic imaging of the abdominal wall is largely underused in clinical practice. The few existing studies have focused on the detection of abdominal wall adhesions after surgery ${ }^{6-8}$. But the quantification of movement and deformations of abdominal muscles could bring valuable information in medical diagnosis and potentially shed light to better understand functional decline in certain neuromuscular diseases or any situations involving abdominal muscle deficiency such as hernia, post-delivery recovery, muscle dystrophy, obesity, muscular fat infiltration and post-mechanical ventilation recovery. The central role of the abdominal wall in visceral restraint, intra-abdominal pressure regulation ${ }^{9}$, posture ${ }^{10,11}$ and breathing 12 may warrant a stress diagnosis in a significant number of situations. However no post-processing method for the quantification of abdominal muscles deformations has been reported so far. Accurate deformation quantification necessitates proper segmentation throughout the dynamic image series, which stands as the primary technical challenge to achieve reliable in vivo biomechanical evaluation. The large and rapid deformations of the abdominal muscles, the movement of the visceral contents at the vicinity of the muscles and the potential imaging artifacts make the segmentation of subtle abdominal muscles particularly complex.

Nevertheless, MRI is an imaging modality of choice for the dynamic evaluation of abdominal wall soft tissues deformation. Complementary to clinical examination, MRI can offer an in-depth analysis of abdominal muscles ${ }^{13}$. On the contrary to ultrasound imaging, MRI can be used to assess the entire abdominal wall and can offer a continuous dynamic analysis during abdomen movements. Although MRI displays a large panel of advantages, the medical community is still lacking a standardized MRI protocol coupled with post-processing methods which would provide accurate and quantitative information that could be used by clinicians.

In the present study, we intended to develop, validate and assess a semi-automatic postprocessing method dedicated to real-time dynamic MRI aiming at a fast and reliable quantification of abdominal wall muscles deformations during a controlled breathing exercise. A coarse feature tracking step allowed the selection of the inhalation cycle of maximum abdominal excursion. Over this image series, the described method combines i) a supervised $2 \mathrm{D}+\mathrm{t}$ segmentation procedure of the abdominal wall muscles, ii) the quantification of muscles deformations based on masks registration and iii) the mapping of deformations within muscle sub-zones leveraging a dedicated automatic parcellation. 


\section{Subjects and methods}

The study was approved by the ethics committee (IDRCB: 2019-A00806-51) and was conducted according to national legislation on interventional research and the declaration of Helsinki.

\subsection{Subjects}

Ten healthy subjects $(33.5 \pm 10.8$ y.o., 5 females) were scanned after they provided their informed written consent. Information related to their physical activity and smoking status was gathered. A score related to physical activity was defined as high (at least 3 to 4 exercise sessions a week), moderate (from 1 to 2 exercise sessions a week) or mild (less than 1 exercise session a week).

\subsection{MRI protocol and breathing exercise}

The abdominal region of the participants was imaged using a 3T MRI scanner (MAGNETOM Verio, Siemens Healthineers, Erlangen, Germany) with a torso-dedicated 32 channels array coil. Subjects were supine and axial images with T1/T2 contrast from a single slice located at the L3L4 disc level were recorded repeatedly with a time resolution of $182 \mathrm{~ms}$ using a cine-bSSFP sequence (echo train duration for 1 single-shot image: 182ms, TE/TR: $1.4 / 3.1 \mathrm{~ms}$, flip angle: $50 \mathrm{deg}$, field of view: $360 \times 360 \mathrm{~mm} 2$, pixel size: $2.3 \times 1.7 \mathrm{~mm} 2$, slice thickness: $8 \mathrm{~mm}$, GRAPPA 4 with 24 reference lines).

Subjects performed four complete controlled breathing cycles guided by an audio signal. Instructions were given to breathe as regularly as possible trying to get maximal abdominal amplitude in both inhalation and exhalation phases. An initial training session was performed before the MRI session on an examination table as many times as necessary to get used to the imposed frequency (1 cycle / 5s). Given the $182 \mathrm{~ms}$ echo train duration, around 27 images were recorded during each cycle from end-exhalation to end-inhalation.

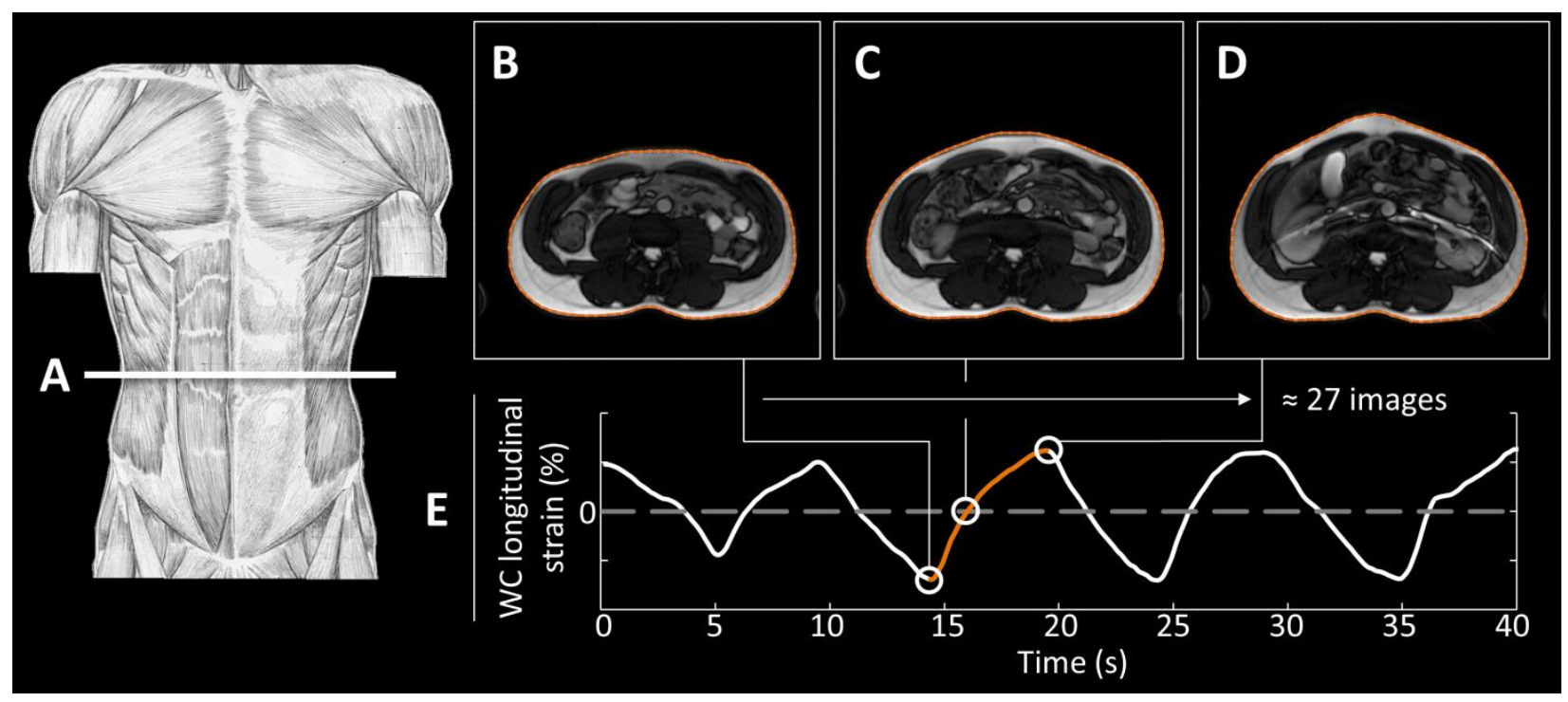

Figure 1 : A : position of the MRI slab i.e. (L3-L4); Axial images of the abdomen recorded at endexhalation (B), at rest (C) and at end-inhalation (D); in each image, the waist circumference (WC), 
highlighted in orange, was automatically tracked by the feature tracking algorithm; E: Typical WC longitudinal strain time-dependent changes during four breathing cycles. The zero-strain corresponds to the abdomen at rest. The orange line indicated the corresponding changes during the 2 nd inhalation which is the inhalation cycle of maximum abdominal excursion.

\subsection{Image selection}

An image dataset of 247 images was acquired for each subject during the $45 \mathrm{~s}$ of controlled breathing. A semi-automatic feature tracking (FT) algorithm based on spatial correlation was developed using Matlab (Matworks, 9.4 R2018a, Natick, MA) as previously reported for the left atrial function 4 . On that basis, inhalation and exhalation phases were distinctly isolated during the controlled breathing exercise (figure 1) in order to select the MR images of interest for this study. The details of the FT algorithm are as follows:

(i) A manual delineation of the abdominal circumference was performed on the median slice of a given image dataset. Then 85 equidistant markers were interpolated over the contour using a Canny edge detection method (sensitivity threshold $=0.2)^{14}$.

(ii) A feature tracking of the 85 markers was performed on the whole image dataset. For each marker, a $3 \times 3$ pixels ROI was centered on the marker's position and a 2D correlation was used to track similar anatomic features on analogous ROIs localized on the neighboring images. The highest correlation was selected as an indicator of the new position of the marker on the neighboring image. The method was designed to avoid the sliding of the markers along the border between the subcutaneous fat and the air which delimits the abdomen on the images.

The length of the reference waist circumference (WC) $l_{0}$ was measured from another MRI dataset acquired during a breath-hold session performed at the end of a smooth exhalation while the abdominal wall was as relaxed as possible (i.e. resting position). At each time step, the measurement of the abdominal circumference $l_{t}$ was defined as the length of the segmented closed line passing through the 85 markers. The WC longitudinal strain $S(t)$ was defined as the temporal variation of the WC length as follows:

$$
S(t)=\frac{l_{t}-l_{0}}{l_{0}}
$$

where $l_{0}$ is the length of the WC at rest, $l_{t}$ refers to the length at time $t$.

A typical WC longitudinal strain curve during the whole sequence is displayed in figure 1 . The maximum and the minimum strain peaks corresponding to the end-exhalation and endinhalation phases respectively were calculated from the longitudinal strain curve. The inhalation amplitude was calculated as the difference between successive minimum and maximum strain peaks.

For each subject, the MR slices corresponding to the greatest amplitude inhalation (i.e. maximum abdominal excursion) were isolated. Those MR slices are referred to as "volume of interest" as visible in figure 2 (section A). The first and last slices of each volume of interest were related to the end-exhalation and end-inhalation phases respectively. 


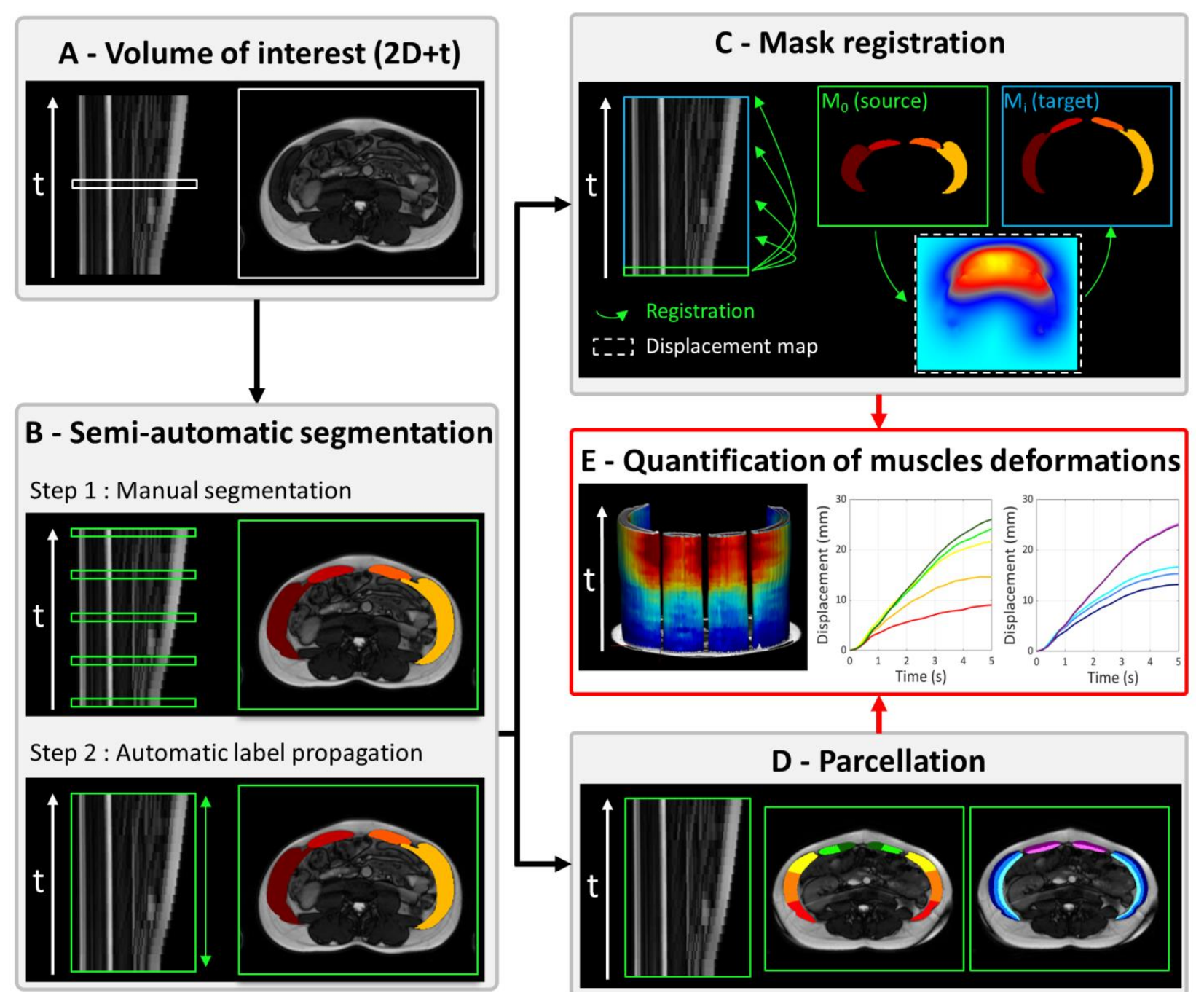

Figure 2: Quantification of muscles deformations pipeline; $A: 2 D+t$ volume of interest in sagittal view with an arbitrary intermediate axial slice; $B$ : the number of manually segmented slices as well as the parameters for automatic label propagation are the result of a comparative analysis visible in supplementary material.

\subsection{Semi-automatic segmentation}

\subsubsection{Manual segmentation}

As a prerequisite, a N4 bias inhomogeneity correction was performed on the MR images volumes of interests as previously described 15 . As illustrated in figure 2 , four regions of interest (ROI) were identified throughout the inhalation cycle. These regions were the right and left rectus abdominis (RRA-LRA) and the right and left lateral abdominal muscles (RLM-LLM). The three lateral abdominal muscles or flat muscles (external oblique, internal oblique and transversus abdominis) were considered as a single ROI (RLM-LLM) given the images resolution. Manual segmentation was performed using FSLeyes, the image viewer included in the FSL toolbox16, with the help of a homemade FSL-embeded plugin ("Superseg" International Patent \# PCT/FR2018/051732 related to the Supervised Segmentation of individual muscles in MR Images). The "Superseg" plugin integrates a contour delineation tool 
based on the selection of control points and interpolation using cubic Catmull-ROM spline curves. MR image volumes were manually segmented by an expert (AJ) on five slices as visible in figure 3.

In addition, MRI volumes of 5 arbitrarily selected subjects (total of 127 slices) were fully manually-segmented to be used as the ground truth for the validation purpose of the proposed semi-automatic segmentation.

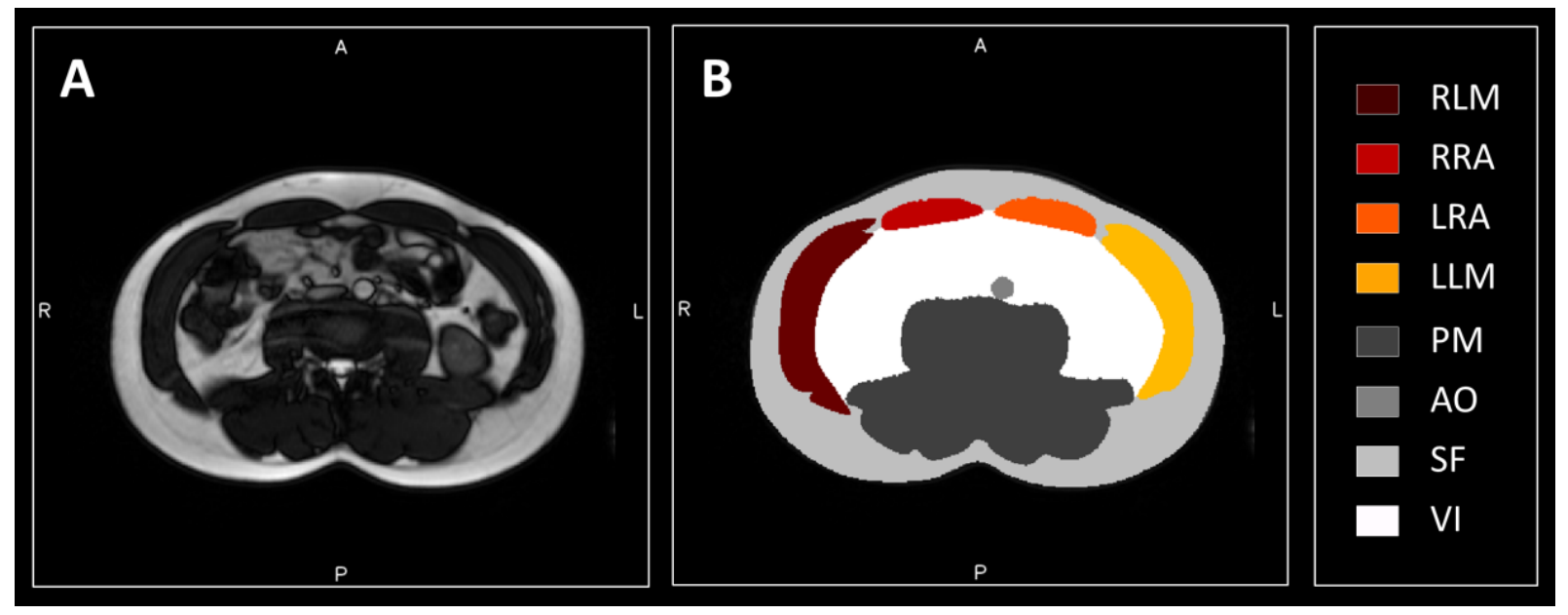

Figure 3 : Typical MR image recorded in the axial plane of the abdomen (A) with the corresponding regions of interest (B); $R L M$ : right lateral muscles, $R R A$ : right rectus abdominis, LRA : left rectus abdominis, LLM : left lateral muscles, PM : posterior abdominal muscles, AO : aorta, SF : subcutaneous fat, VI : viscera.

\subsubsection{Automatic label propagation}

The five segmented masks were propagated to the remaining slices of the volume of interest $(2 \mathrm{D}+\mathrm{t})$ using an automatic label propagation algorithm as previously described 17 (figure 2, section B). This algorithm implemented with the ANTs library ${ }^{18}$, consisted of a registration process with rigid and affine transformations, followed by a diffeomorphic multi-level registration with B-Spline regularization. The choice of the propagation strategy and the registration parameters is the result of a comparative analysis visible in the supplementary materials.

The resulting segmentation was compared to the ground truth i.e. the entire manual segmentation of the 4 abdominal wall muscles (RRM, RRA, LRM, LRA) based on the computation of Dice similarity coefficient (DSC) and the Hausdorff distance (HD) which were calculated for the automatically-segmented slices thereby excluding the manually-segmented slices.

\subsubsection{Morphometric measurements}

From the segmented masks of the abdominal wall ROIs, changes in muscle area, elongation and eccentricity metrics were quantified throughout the inhalation period. Those metrics were measured using the LabelGeometryMeasures command implemented in the Ants Library ${ }^{19}$. 
Elongation was defined as:

Eccentricity was defined as:

$$
\text { elongation }=\frac{\text { MajorAxisLength }}{\text { MinorAxisLength }}
$$

$$
\text { eccentricity }=\frac{\sqrt{\text { MajorAxisLengt }{ }^{2}-\text { MinorAxisLengt }^{2}}}{\text { MajorAxisLength }}
$$

Eccentricity near 0 refers to a shape approaching a circle (elongation 1 ) whereas eccentricity near 1 refers to a shape approaching a line (elongation $\sim+\infty)$.

\subsection{Individual muscle parcellation}

In order to discriminate muscles areas, a dedicated automatic parcellation of the segmented masks was performed according as previously described ${ }^{20}$. A polar coordinate system was used to determine the position of each voxel of the segmented masks and the localization system was based on the mass center of the lateral muscles (RLM, LLM). On that basis, lateral muscles ROIs (RLM, LLM) were automatically split into three depth (superficial, intermediate, and deep layers) and three angular levels (posterior, central and anterior) as illustrated in Figure 4 . The three layers were intended to mimic the three anatomically described muscle layers i.e. external oblique, internal oblique and transversus abdominis. The rectus abdominis muscles ROIs (RRA, LRA) were split into two depths (superficial and deep) and two angular (medial and lateral) levels. 

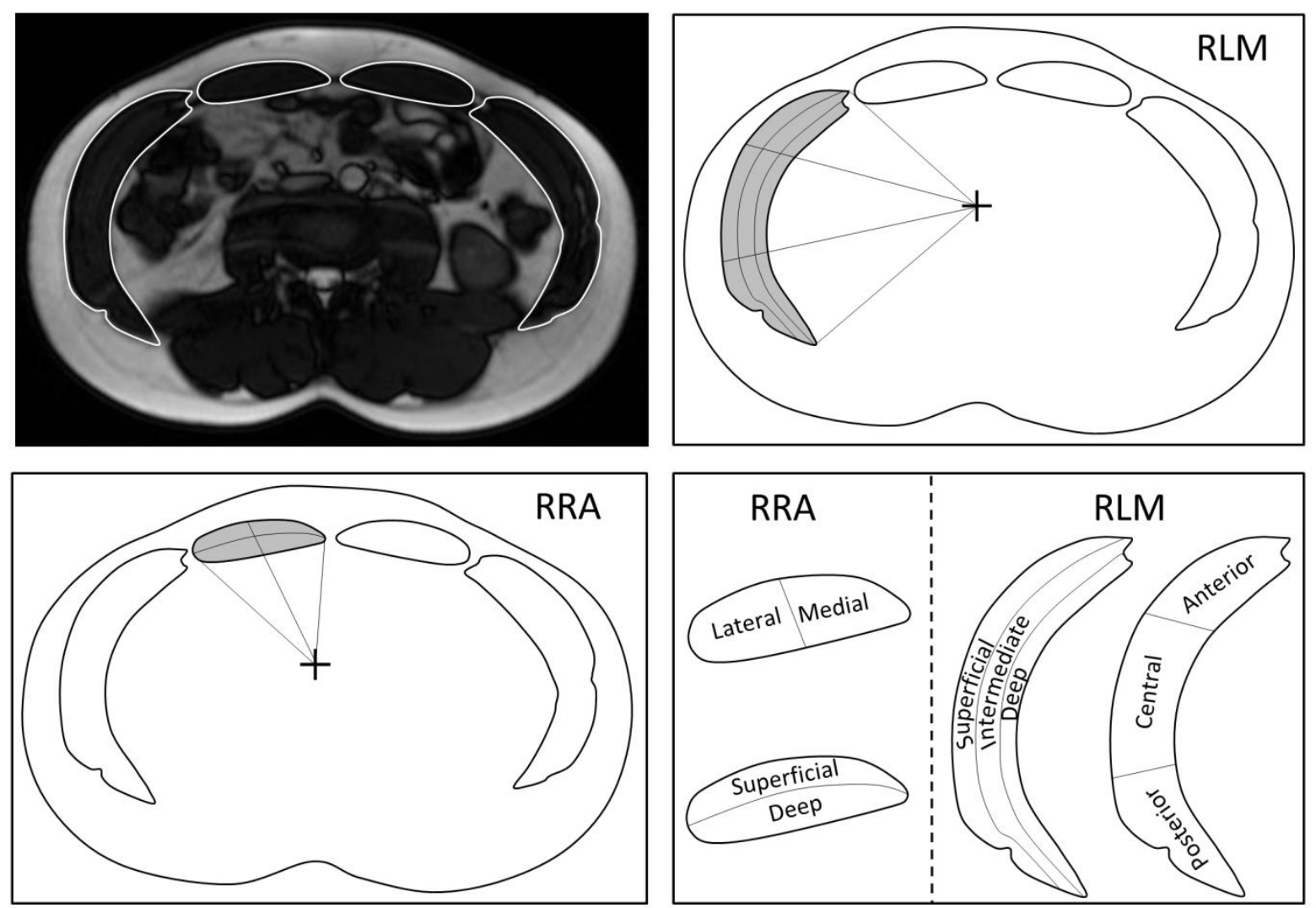

Figure 4: Abdominal wall muscles ROIs parcellation; Example of the specific parcellation of right lateral muscles (RLM) and of the right rectus abdominis (RRA)

\subsection{Quantification and time-dependent changes of muscles deformations}

The evolution over time of the displacement magnitude of the abdominal wall muscles compared to their initial position (i.e. end-exhalation) was computed from registration of the segmented masks as illustrated in figure 2 (section $C$ ). For each dataset of $n$ masks, the first segmented mask $M_{0}$ was considered as the single source while $M_{i}(1 \leq i \leq n)$ was considered as the set of target segmented masks. Consecutive registrations were performed between $\mathrm{M}_{0}$ and $\mathrm{M}_{\mathrm{i}}$ using a Time Varying VelocityField transformation model implemented in the Ants Library and recommended for large deformations. The parameters for this registration stage were: gradient step $=2.0$, cost function as mean squares, 5 multiresolution steps with, respectively, $1200 \times 1200$ $\times 100 \times 20 \times 0$ iterations max per level, shrink factor $=8 \times 6 \times 4 \times 2 \times 1$, and smoothing sigma $=8$ $\times 6 \times 4 \times 2 \times 1$ voxel. The displacement magnitudes between the source segmented mask $\mathrm{M}_{0}$ and each successive segmented mask $M_{i}$ were then generated at pixel scale using a script implemented in the Ants library that calculates displacement amplitudes from transformation fields (i.e. warps) using Euclidean distance (antsdeformationmag.sh).

The validity of the $M_{0}$ to $M_{i}$ segmented masks registration, and consequently of the displacement magnitude calculation was evaluated using the DSC and HD metrics computed between the registered (i.e. warped) $M_{0}$ segmented mask and the corresponding target $M_{i}$ segmented mask. This validation step was carried out for the 10 volunteers for all consecutive 
registrations $(n=242)$. The similarity coefficients (DSC and HD) were averaged for all 4 abdominal wall muscles ROI (RLM, RRA, LRA, LLM).

The temporal evolution of the average displacement of the muscular regions was calculated within each previously defined parcels.

\section{Results}

\subsection{Demographic data}

The demographic data of the cohort are presented in Table 1. The physical activity score was variable within the group.

Table 1 : Demographic data

\begin{tabular}{|c|c|c|}
\hline Women / Men (n) & $5 / 5$ & \\
\hline Age $(y r)^{*}$ & $33.5 \pm 10.8$ & $22.0 / 54.0$ \\
\hline Body mass index $\left(\mathrm{kg} / \mathrm{m}^{2}\right)^{*}$ & $22.7 \pm 2.5$ & $17.5 / 26.6$ \\
\hline Waist circumference $(\mathrm{mm})^{*}$ & $814 \pm 75$ & $678 / 921$ \\
\hline Physical activity score (High/Moderate/Mild) & $3 / 6 / 1$ & \\
\hline Non-smokers / Smokers (n) & $8 / 2$ & \\
\hline
\end{tabular}

* values presented as mean $\pm S D$ min $/ \max$

\subsection{Image selection}

For each subject, the MR slices corresponding to the inhalation with the largest longitudinal strain amplitude were isolated. Overall, 230 slices were integrated into the rest of the study. The duration of the selected inhalation bout varied from $3.6 \mathrm{~s}$ to $5.3 \mathrm{~s}$ and the corresponding longitudinal strain increased regularly over time (figure 5 , all curves aligned to a 5 s scale). The mean longitudinal strain was $6.1 \pm 2.0 \%$. 


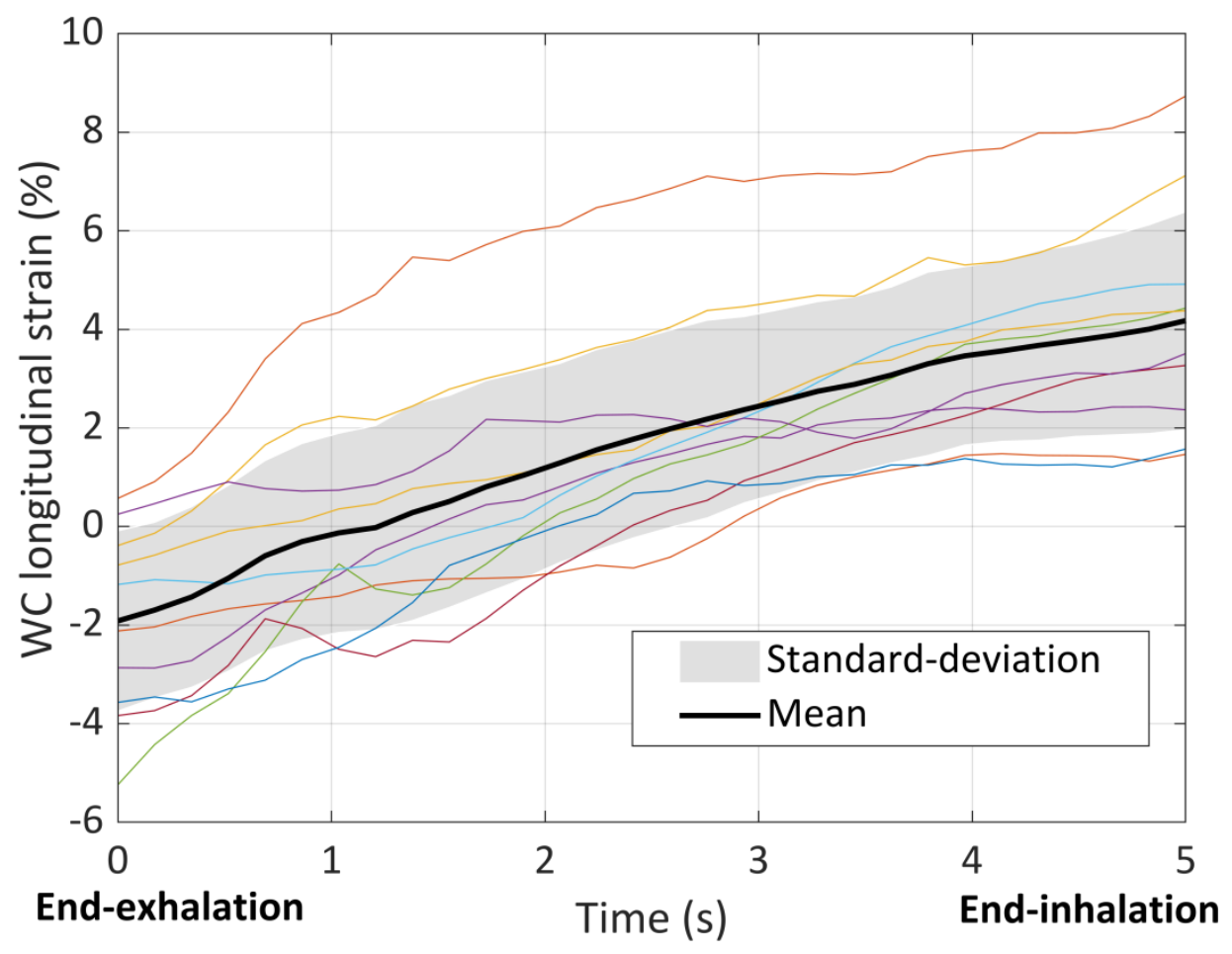

Figure 5 : Waist circumference (WC) longitudinal strain during the inhalation cycle of maximum abdominal excursion for the 10 subjects.

\subsection{Semi-automatic segmentation}

\subsubsection{Performances}

Typical manual and automatically-segmented masks are visible in figure 6. The DSC and HD metrics computed between the automatically-segmented slices and ground truth segmentation were respectively $0.95 \pm 0.03$ and $1.9 \pm 1.1 \mathrm{~mm}$. The DSC scores for RLM, RRA, LRA and LLM muscles were $0.96,0.94,0.93$ and 0.96 respectively. The corresponding HD values were 1.91, $1.81,1.85$ and $2.03 \mathrm{~mm}$ respectively. It is noteworthy that the segmentation of the Rectus Abdominis muscle was less accurate in terms of DSC scores as compared to the lateral muscles. The average time required for the manual delineation of the five input slices was $12 \mathrm{~min} 30 \mathrm{~s}$. In comparison the complete manual segmentation required about $65 \mathrm{~min}$. On that basis, $80 \%$ of the manual segmentation time could be spared.

\subsubsection{Muscle area and geometry changes}

Time-dependent changes in muscle area, elongation and eccentricity are illustrated in figure 6 over a 5 s timescale. The patterns and quantitative changes were very similar between the right and left sides. The lateral muscles show the largest area and eccentricity changes during inspiration. At end of the exhalation session, the lateral muscles area (Right: $27.1 \pm 3.8 \mathrm{~cm}^{2}$; Left $: 27.1 \pm 4.5 \mathrm{~cm}^{2}$ ) was around 4 times larger than the Rectus Abdominis muscles area (Right: $7.1 \pm$ $1.6 \mathrm{~cm}^{2}$; Left : $\left.6.7 \pm 1.6 \mathrm{~cm}^{2}\right)$. The lateral muscles area decreased almost linearly during inhalation (area at end-exhalation; Right : $26.1 \pm 3.8 \mathrm{~cm}^{2}$; Left : $25.7 \pm 4.3 \mathrm{~cm}^{2}$ ) whereas the 
overall area of the rectus muscles remained constant (area at end-exhalation ; Right : $7.0 \pm 1.3$ $\mathrm{cm}^{2}$; Left : $6.5 \pm 1.5 \mathrm{~cm}^{2}$ ).

The elongation of the Rectus Abdominis muscles (Right : $4.9 \pm 1.2$; Left : $5.0 \pm 1.4$ at endexhalation) was larger than that of the lateral muscles (Right : $2.4 \pm 0.4$; Left : $2.5 \pm 0.3$ at endexhalation). The elongation of the lateral muscles increased between end-exhalation and endinhalation (Right: $2.9 \pm 0.4$; Left : $2.9 \pm 0.4$ at end-inhalation). A similar increase was observed for the Rectus abdominis muscles (Right : $5.3 \pm 1.3$; Left : $5.5 \pm 1.6$ at end-inhalation). However, changes were "noisier", likely as a result of a larger inter-subject dispersion.

The eccentricity of the Rectus Abdominis muscles (Right : $0.98 \pm 0.01$; Left : $0.98 \pm 0.01$ at endexhalation) was also larger than that of the lateral muscles (Right : $0.90 \pm 0.03$; Left : $0.91 \pm 0.03$ at end-exhalation). The eccentricity of the lateral muscles increased between end-exhalation and end-inhalation (Right : $0.93 \pm 0.02$; Left : $0.93 \pm 0.02$ at end-inhalation) whereas the Rectus abdominis muscles kept a constant eccentricity throughout the whole inhalation (Right : $0.98 \pm$ 0.01 ; Left : $0.98 \pm 0.01$ at end-inhalation).

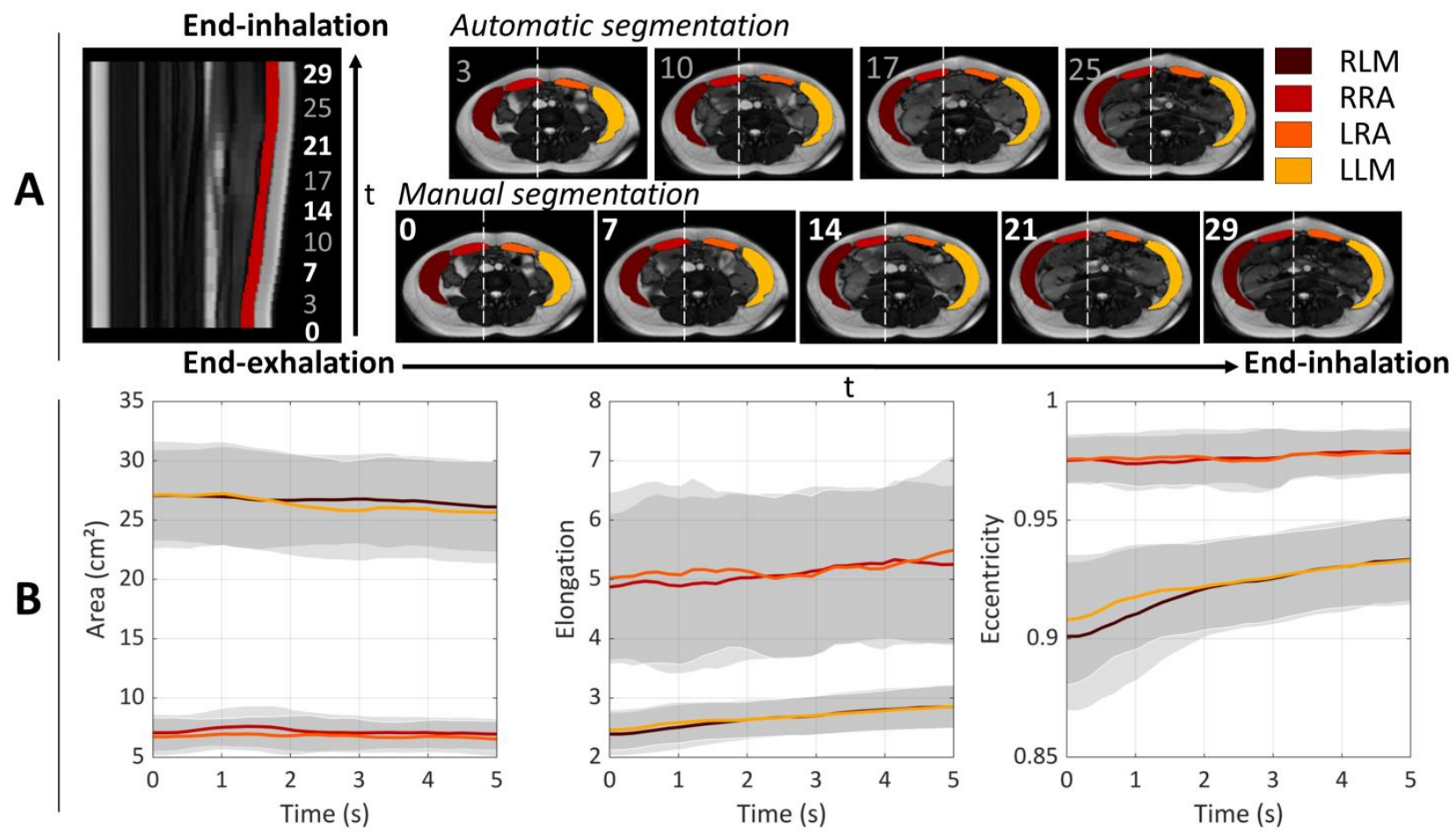

Figure $6:$ A : Left : Sagittal view of the 2D+t volume of interest; Right: Typical manual and automatic segmentation masks, the white dashed line indicates section location of the sagittal view; B : Muscle geometry metrics evolution during inhalation; From left to right : area, elongation and eccentricity.

\subsection{Quantification and time-dependent changes of muscles deformations}

\subsubsection{Validation}

The DSC and HD metrics computed between the registered (i.e. warped) $\mathrm{M}_{0}$ segmented mask and the corresponding target $M_{i}$ segmented mask were respectively $0.98 \pm 0.01$ and $2.1 \pm 1.5$ $\mathrm{mm}$. 


\subsubsection{Magnitude of displacement maps}

The maps related to the magnitude of displacement resulting from mask registration are presented in Figure 7 for a typical subject i.e. for whom the corresponding values were close to the mean values of the cohort.

For each muscle, the set of $(2 \mathrm{D}+\mathrm{t})$ masks was merged to one single contiguous 3D mask and a 3D mesh surface was reconstructed using Brainvisa 4.6 software (AimsMesh command, lowpass smoothing method with 500 iterations). The corresponding 3D magnitude maps were projected on 3D meshes with fusion algorithm implemented in Anatomist viewer (Brainvisa 4.6 Software). The fusion method consisted in averaging the neighbor values included in a sphere of $5 \mathrm{~mm}$ radius, centered at each vertex of the mesh.

These 2D and 3D maps were used in order to quantify the magnitude of displacement changes of the abdominal muscles from the end-exhalation to end-inhalation and for the whole set of intermediate states. During inhalation, the displacement magnitude of each muscle group gradually increased and reached a maximum at end of the inhalation phase. In the axial plane, the magnitude of displacement within the rectus abdominis muscles was quite homogeneous. On the contrary, for the lateral muscles, the magnitude of displacement of the posterior part did not exceed $4 \mathrm{~mm}$ during the entire inhalation whereas a $25 \mathrm{~mm}$ change was quantified in the anterior part and more particularly in the internal part. The displacements of the internal faces of the lateral muscles were larger than those of the external faces.

B

2D Axial views

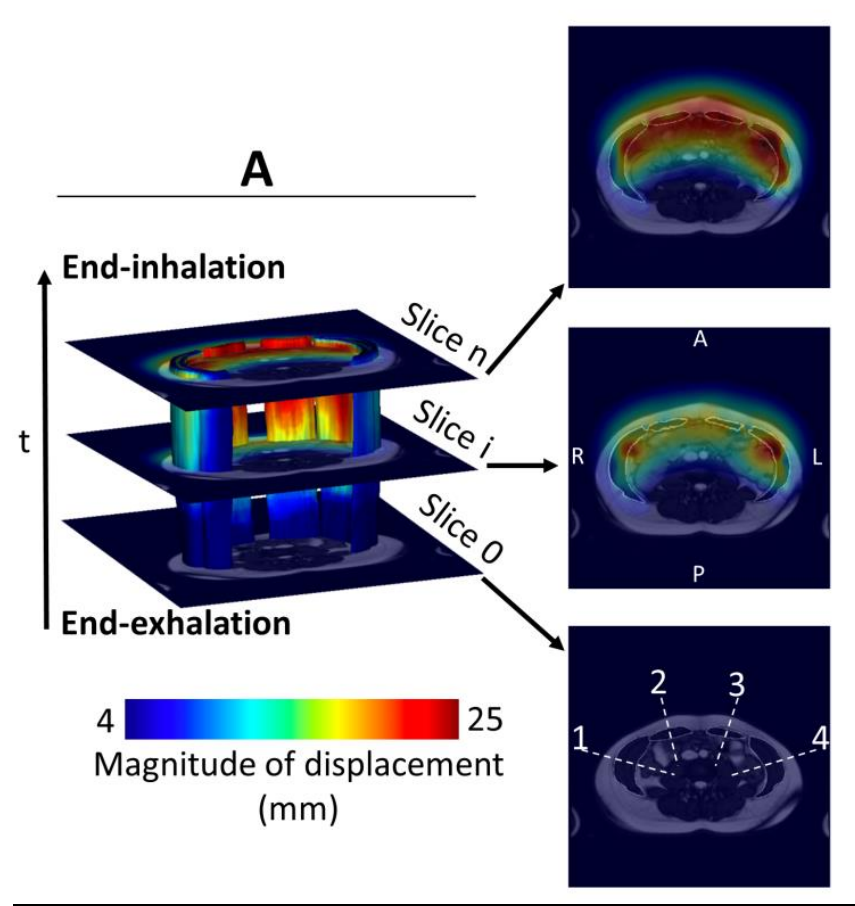

C 3D Inside views
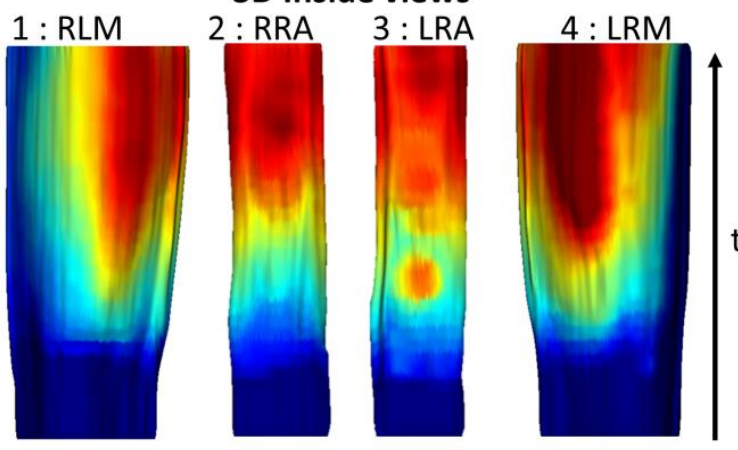

3D Outside views
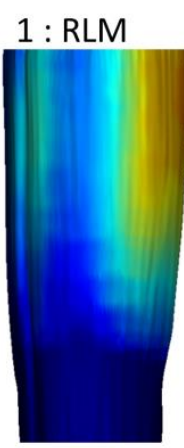

2 : RRA

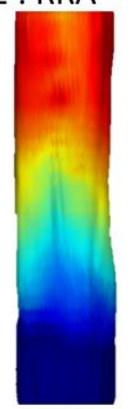

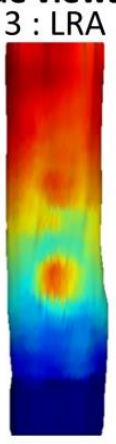

Figure 7 : Typical magnitude of displacement maps. These maps were obtained for the subject closest to the mean values of the cohort; $A: 3 d$ general view of displacement magnitude within the abdominal wall muscles from end-exhalation to end-inhalation with three anatomical MRI slices; $B: 2 D$ axial views of the 
magnitude of displacement computed on the three previous anatomical MRI slices; $C: 3 D$ visualization of the magnitudes within each individual muscle group.

\subsubsection{Magnitude of displacement within parcels}

From the maps previously described (3.4.2), time dependent change of the average displacement value within each parcel has been calculated. The average results for the whole cohort are shown in figure 8.

The magnitude of displacement evolved initially linearly and then was gradually stabilized towards the end of inhalation. The maximum value was reached at end-inhalation and the largest displacements occurred for the medial RA parcel $(26.1 \mathrm{~mm}$ at end-inhalation). The smallest displacements were localized in the posterior parcel of the LM $(9.0 \mathrm{~mm}$ at endinhalation). For the rectus abdominis, both deep and superficial parcels displayed a similar displacement (25.3 $\mathrm{mm}$ and $25.0 \mathrm{~mm}$ at end-inhalation respectively). A larger difference was quantified between the medial and the lateral parcels $(26.1 \mathrm{~mm}$ and $24.2 \mathrm{~mm}$ at end-inhalation respectively). The anterior, central, and posterior parcels of the LM showed large differences (21.6 $\mathrm{mm}, 14.6 \mathrm{~mm}$ and $9.0 \mathrm{~mm}$ at end-inhalation respectively). The deep, intermediate, and superficial layers displayed relatively similar displacements $(16.7 \mathrm{~mm}, 15.4 \mathrm{~mm}$ and $13.2 \mathrm{~mm}$ at end-inhalation respectively). 


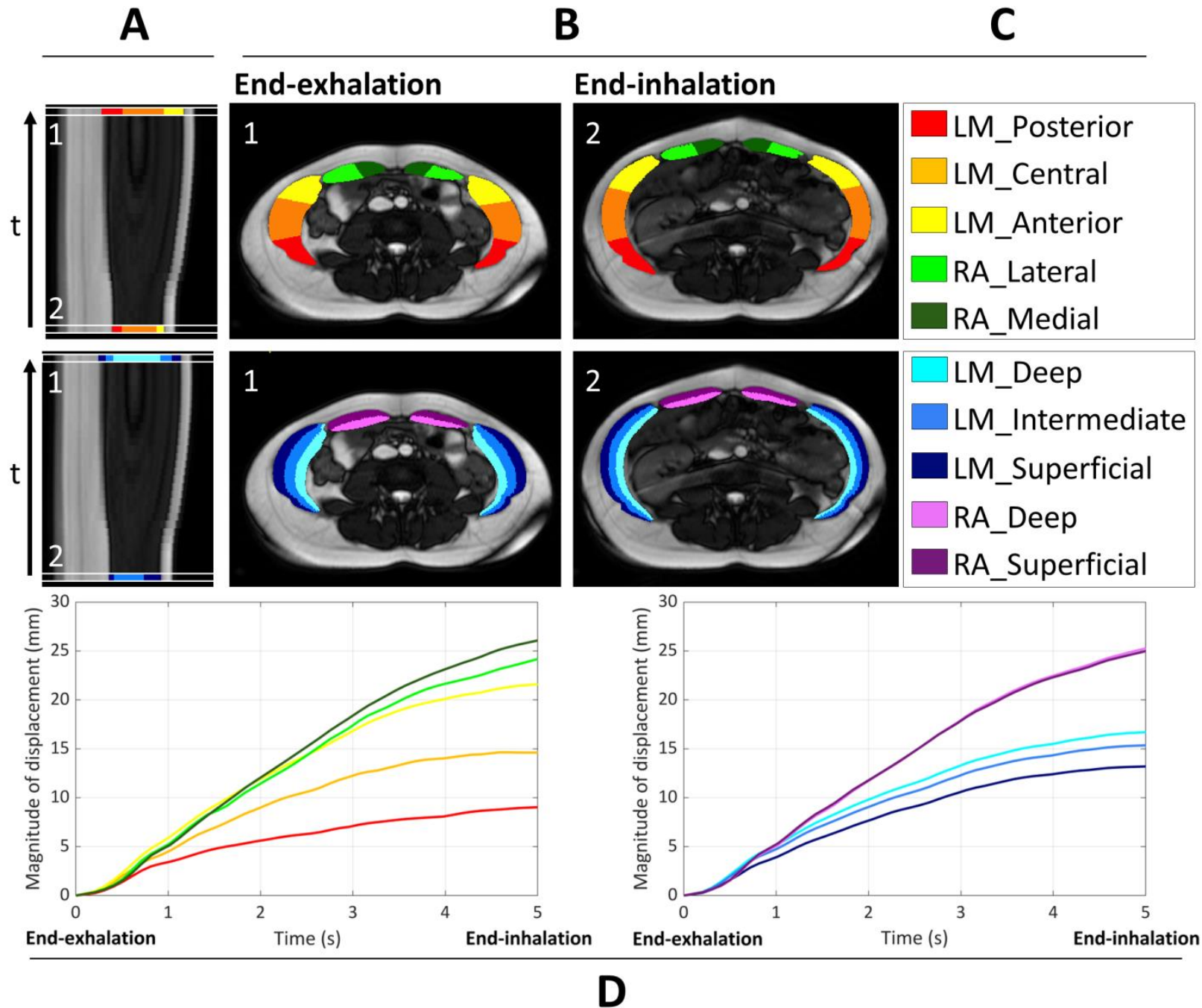

Figure 8 : A : Sagittal 3D view $(2 D+t) ; B$ : Typical parcellation at end-exhalation and end-inhalation; $C$ : Parcellation nomenclature; $D$ : Curves representing the mean magnitude of displacement over the time, for the whole group. 


\section{Discussion}

In this study, we designed a dedicated semi-automatic post-processing method based on features tracking, optimized $2 \mathrm{D}+\mathrm{t}$ semi-automatic segmentation, segmented mask registration and automatic parcellation. Applied on cine-BSSFP MRI data, this method allowed to quantify and localize spatially and temporally the abdominal wall muscles deformations during breathing. An exhaustive quantitative picture of abdominal muscles deformations based on global metrics of shape and geometry linked to localization aspects was reported.

The accuracy of the semi-automatic segmentation procedure was supported by a very high DSC $(0.95 \pm 0.03)$ together with a very low $\mathrm{HD}(2.3 \pm 0.7 \mathrm{~mm})$. As a matter of comparison, DSC values for a fully automated multi-atlas-based segmentation of cardiac muscle based on spatiotemporal registrations ranged from 0.88 to $0.94^{21}$. DSC values for semi-automated cardiac muscles segmentation reached 0.90 using neural-networks-based segmentation 22 and 0.89 with snake deformable models ${ }^{23}$. Automatic muscle segmentation method reached DSC $=0.87 \pm 0.11$ for individual thigh muscles, using multi-atlas-based approach ${ }^{24}$. These results have to be considered in the light of the 0.96 DSC value which has been reported for a manual segmentation performed by two experts ${ }^{17}$. On that basis, the present results related to the semiautomated abdominal wall muscles segmentation illustrated a very high segmentation performance. Our semi-automatic segmentation strategy required the manual delineation of $20 \%$ of the data. For future applications, taking into account the quality of the segmentation, it seems possible to reduce this manual segmentation time so that the clinical utilization could be easier. The robustness of the displacement magnitude calculation was also supported by a very high DSC $(0.98 \pm 0.01)$ together with a very low HD $(2.1 \pm 1.5 \mathrm{~mm})$ computed between the registered (i.e. warped) $\mathrm{M}_{0}$ segmented mask and the corresponding target $\mathrm{M}_{\mathrm{i}}$ segmented mask. These scores indicated a near-perfect mask registration despite the large abdominal wall muscle deformations associated with inhalation. Finally, the automatic parcellation had been used in previous studies in order to discriminate areas within different organs such as heart 25,26 brain ${ }^{27-29}$ and muscles ${ }^{20}$ but, to our knowledge, was never applied to abdominal wall muscles. In the present study, it enabled the distinction of muscle substructures which could be anatomicallyrelevant but could not be distinguished based on images contrast.

Our results did not only support the specificity of muscle involvement during breathing but genuinely provided some quantitative supports related to the deformation of abdominal muscles during respiration. Previous studies have only reported external measurements conducted at the abdomen surface ${ }^{30,31}$. Our results could be partially compared to those reported using three-dimensional stereography during maximum excursion ${ }^{30}$ and insufflation for laparoscopic surgery ${ }^{31}$. Although the corresponding mechanical stress was different, the deformations distribution we reported during inhalation was similar with deformations concentrated on the central zone of the abdominal wall. During a $12 \mathrm{mmHg}$ abdomen inflation, Song et al. ${ }^{31}$ reported a maximum $40 \mathrm{~mm}$ displacement located at the center of the abdomen wall. This result is comparable to the mean magnitude of displacement computed in the medial part of the rectus abdominis at end-exhalation $(26.0 \pm 9.1 \mathrm{~mm}, \max =44.0 \mathrm{~mm})$. This displacement distribution can be supported by anatomical observations. During inhalation the central part of the abdominal wall is expected to be more deformable given that it is located far from the areas of muscular insertions on the bones which form the rigid frame of the abdominal 
wall (iliac crests, pubic symphysis, ribs and spine) while the posterior part of the lateral muscles is located close to the insertion of the transverse muscle on the lumbar vertebrae.

The WC increase we quantified during inhalation on the basis of the features tracking method is likely related to the increased intra-abdominal pressure resulting from the diaphragm contraction and the corresponding increased abdominal volume previously reported 32 . Considering that the muscle area changes are tightly linked to the contraction-relaxation process $^{33}$, our results suggest that the lateral muscles are contracting during forced exhalation, relaxing during inhalation whereas the rectus abdominis muscles would be minimally active. This is further supported by the fact that shape and area changes were larger for lateral muscles during inhalation as compared to rectus abdominis muscles and by the fact that the displacement values were very similar between each rectus abdominis parcel and very different between lateral muscles parcels. These observations agree with the basic physiological knowledge regarding respiratory effort 32,34 . It has been reported using surface and intramuscular electromyography that the transversus abdominis and the internal oblique muscles (i.e. muscles of the lateral muscles group) were strongly activated at the end of forced exhalation whereas the rectus abdominis muscles were very slightly activated 11 . This confirms why the magnitudes of displacement of the deep and intermediate parcels of the lateral muscles are greater than that of the superficial parcel (i.e. location of the external oblique muscle).

Several limitations have to be acknowledged for the present study. 2D dynamic MRI might be considered as limited given that displacements of organs of interest in the direction perpendicular to the section plane cannot be assessed. However, it can be reasonably assumed that abdominal muscles displacements in the cranio-caudal direction are negligible and so because the muscular insertions on the bone rigid frame are likely to constrain the movements in the cranio-caudal direction. As additional evidence and as illustrated in the supplementary materials, we assessed the displacements from a $2 \mathrm{D}+\mathrm{t}$ sagittal dataset and found a ratio of $1 / 20$ between the displacements in the axial plane (i.e. imaged plane) and those in the sagittal plane. The rationale behind the choice of a single slice was related to the fact that we intended to reach a high temporal resolution. Such a resolution was necessary in order to properly assess the exercise dynamics. A 3D dynamic MRI could be considered. However, this would imply a reduced temporal resolution so as to keep the same spatial resolution. As the reduced temporal resolution should be related to larger deformations between successive MR acquisitions, the present semi-automatic segmentation is not expected to be effective. It is noteworthy that the choice of a given plane for dynamic MRI acquisition has become a standard practice in clinical MRI5.

It could be argued that the demographic data of our relatively young cohort do not coincide with those of patients with abdominal muscle deficiency. Although, we clearly proved the effectiveness and interest of the method in a group of healthy volunteers, the method should also be operational in subjects with various histological changes such as muscle atrophy and fat infiltration inasmuch as the initial segmentation step is manual. In addition, our cohort was composed of healthy subjects ranging from 24 to 54 years old and a balance between men and women. On that basis, the method was efficient for people with various anthropometric characteristics and with a BMI ranging 17.5 to 26.6 i.e. underweight to overweight. 
The methodology we reported in the present study could be used to further understanding the physiology and biomechanics of the abdominal wall in a variety of exercises involving motion and contraction of the abdominal wall. It could also provide a quantitative frame for the assessment of dysfunctions of the abdominal wall mobility.

\section{Acknowledgements}

We would like to thank Claire Costes, Lauriane Pini and Patrick Viout (CRMBM/CEMEREM UMR CNRS 7339) for their help in integrating the volunteers and carrying out the MRI acquisitions, Thomas Troalen (Siemens Healthineers) for his assistance in the optimization of the MRI sequences and Morgane Evin from (Gustave Eiffel University) for her help and insightful comments in the development of the features tracking algorithm.

\section{Fundings}

This research was supported by IFSTTAR (French Institute of Science and Technology for Transport, Development and Networks), CNRS (Centre National de la Recherche Scientifique) and APHM (Assistance Publique Hôpitaux de Marseille).

\section{References}

1. Kim RJ, $\mathrm{Wu}$ E, Rafael A, et al. The Use of Contrast-Enhanced Magnetic Resonance Imaging to Identify Reversible Myocardial Dysfunction. $N$ Engl J Med. 2000;343(20):1445-1453. doi:10.1056/NEJM200011163432003

2. Liu J, Wang Y, Wen Z, et al. Extending Cardiac Functional Assessment with Respiratory-Resolved 3D Cine MRI. Sci Rep. 2019;9(1):11563. doi:10.1038/s41598-019-47869-z

3. Comiter CV, Vasavada SP, Barbaric ZL, Gousse AE, Raz S. Grading pelvic prolapse and pelvic floor relaxation using dynamic magnetic resonance imaging. Urology. 1999;54(3):454-457. doi:10.1016/S0090-4295(99)00165-X

4. Evin M, Cluzel P, Lamy J, et al. Assessment of left atrial function by MRI myocardial feature tracking. J Magn Reson Imaging. 2015;42(2):379-389. doi:10.1002/jmri.24851

5. Jiang Z, Witz J-F, Lecomte- Grosbras $\mathrm{P}$, et al. Multiorgan motion tracking in dynamic magnetic resonance imaging for evaluation of pelvic system mobility and shear strain. Strain. 2017;53(2):e12224. doi:10.1111/str.12224

6. Lang RA, Buhmann S, Hopman A, et al. Cine-MRI detection of intraabdominal adhesions: correlation with intraoperative findings in 89 consecutive cases. Surg Endosc. 2008;22(11):2455-2461. doi:10.1007/s00464-008-9763-9

7. Fenner J, Wright B, Emberey J, et al. Towards radiological diagnosis of abdominal adhesions based on motion signatures derived from sequences of cine-MRI images. Phys Med. 2014;30(4):437-447. doi:10.1016/j.ejmp.2013.12.002

8. Randall D, Joosten F, Ten Broek RP, et al. A novel diagnostic aid for intra-abdominal adhesion detection in cine-MRI: pilot study and initial diagnostic impressions. $\mathrm{Br} J$ Radiol. 2017;90(1077):20170158. doi:10.1259/bjr.20170158 
9. Cobb WS, Burns JM, Kercher KW, Matthews BD, James Norton H, Todd Heniford B. Normal Intraabdominal Pressure in Healthy Adults. J Surg Res. 2005;129(2):231-235. doi:10.1016/j.jss.2005.06.015

10. De Troyer A. Mechanical role of the abdominal muscles in relation to posture. Respir Physiol. 1983;53(3):341-353. doi:10.1016/0034-5687(83)90124-x

11. Neumann P, Gill V. Pelvic Floor and Abdominal Muscle Interaction: EMG Activity and Intraabdominal Pressure. Int Urogynecology J. 2002;13(2):125-132. doi:10.1007/s001920200027

12. Campbell EJM. An electromyographic study of the role of the abdominal muscles in breathing. $J$ Physiol. 1952;117(2):222-233.

13. Wang F-Z, Sun H, Zhou J, Sun L-L, Pan S-N. Reliability and Validity of Abdominal Skeletal Muscle Area Measurement Using Magnetic Resonance Imaging. Acad Radiol. Published online October 28, 2020. doi:10.1016/j.acra.2020.09.013

14. Canny J. A Computational Approach to Edge Detection. IEEE Trans Pattern Anal Mach Intell. 1986;PAMI-8(6):679-698. doi:10.1109/TPAMI.1986.4767851

15. Tustison NJ, Avants BB, Cook PA, et al. N4ITK: Improved N3 Bias Correction. IEEE Trans Med Imaging. 2010;29(6):1310-1320. doi:10.1109/TMI.2010.2046908

16. McCarthy P. FSLeyes. Zenodo; 2019. doi:10.5281/zenodo.3530921

17. Ogier AC, Heskamp L, Michel CP, et al. A novel segmentation framework dedicated to the followup of fat infiltration in individual muscles of patients with neuromuscular disorders. Magn Reson Med. 2020;83(5):1825-1836. doi:10.1002/mrm.28030

18. Tustison NJ, Avants B avants. Explicit B-spline regularization in diffeomorphic image registration. Front Neuroinformatics. 2013;7. doi:10.3389/fninf.2013.00039

19. Padfield D, Miller J. A Label Geometry Image Filter for Multiple Object Measurement. Insight J. Published online August 14, 2008:301.

20. Fouré A, Le Troter A, Guye M, Mattei J-P, Bendahan D, Gondin J. Localization and quantification of intramuscular damage using statistical parametric mapping and skeletal muscle parcellation. Sci Rep. 2015;5(1):18580. doi:10.1038/srep18580

21. Shahzad R, Tao Q, Dzyubachyk O, Staring M, Lelieveldt BPF, van der Geest RJ. Fully-automatic left ventricular segmentation from long-axis cardiac cine MR scans. Med Image Anal. 2017;39:44-55. doi:10.1016/j.media.2017.04.004

22. Upendra RR, Dangi S, Linte CA. Automated Segmentation of Cardiac Chambers from Cine Cardiac MRI Using an Adversarial Network Architecture. Proc SPIE-- Int Soc Opt Eng. 2020;11315. doi:10.1117/12.2550656

23. Constantinides C, Chenoune $\mathrm{Y}$, Kachenoura N, et al. Semi-automated cardiac segmentation on cine magnetic resonance images using GVF-Snake deformable models. MIDAS J. Published online July 26, 2009:678. 
24. Le Troter A, Fouré A, Guye $\mathrm{M}$, et al. Volume measurements of individual muscles in human quadriceps femoris using atlas-based segmentation approaches. Magn Reson Mater Phys Biol Med. 2016;29(2):245-257. doi:10.1007/s10334-016-0535-6

25. Lang RM, Bierig $\mathrm{M}$, Devereux RB, et al. Recommendations for chamber quantification. Eur $J$ Echocardiogr. 2006;7(2):79-108. doi:10.1016/j.euje.2005.12.014

26. Cerqueira MD, Weissman NJ, Dilsizian V, et al. Standardized myocardial segmentation and nomenclature for tomographic imaging of the heart. A statement for healthcare professionals from the Cardiac Imaging Committee of the Council on Clinical Cardiology of the American Heart Association. Int J Cardiovasc Imaging. 2002;18(1):539-542.

27. Rademacher J, Galaburda AM, Kennedy DN, Filipek PA, Caviness VS. Human cerebral cortex: localization, parcellation, and morphometry with magnetic resonance imaging. J Cogn Neurosci. 1992;4(4):352-374. doi:10.1162/jocn.1992.4.4.352

28. Honnorat N, Eavani H, Satterthwaite TD, Gur RE, Gur RC, Davatzikos C. GraSP: geodesic Graphbased Segmentation with Shape Priors for the functional parcellation of the cortex. NeuroImage. 2015;106:207-221. doi:10.1016/j.neuroimage.2014.11.008

29. Tzourio-Mazoyer N, Landeau B, Papathanassiou D, et al. Automated anatomical labeling of activations in SPM using a macroscopic anatomical parcellation of the MNI MRI single-subject brain. NeuroImage. 2002;15(1):273-289. doi:10.1006/nimg.2001.0978

30. Klinge U, Müller M, Brücker C, Schumpelick V. Application of three-dimensional stereography to assess abdominal wall mobility. Hernia. 1998;2(1):11-14. doi:10.1007/BF01207767

31. Song C, Alijani A, Frank T, Hanna GB, Cuschieri A. Mechanical properties of the human abdominal wall measured in vivo during insufflation for laparoscopic surgery. Surg Endosc Interv Tech. 2006;20(6):987-990. doi:10.1007/s00464-005-0676-6

32. Campbell EJM, Green JH. The variations in intra-abdominal pressure and the activity of the abdominal muscles during breathing; a study in man. J Physiol.1953;122(2):282-290.

33. Hides JA, Boughen CL, Stanton WR, Strudwick MW, Wilson SJ. A magnetic resonance imaging investigation of the transversus abdominis muscle during drawing-in of the abdominal wall in elite Australian Football League players with and without low back pain. J Orthop Sports Phys Ther. 2010;40(1):4-10. doi:10.2519/jospt.2010.3177

34. Mesquita Montes A, Baptista J, Crasto C, de Melo CA, Santos R, Vilas-Boas JP. Abdominal muscle activity during breathing with and without inspiratory and expiratory loads in healthy subjects. $J$ Electromyogr Kinesiol. 2016;30:143-150. doi:10.1016/j.jelekin.2016.07.002 